\title{
Carbon nanotube electrodes for electrochemiluminescence biosensors
}

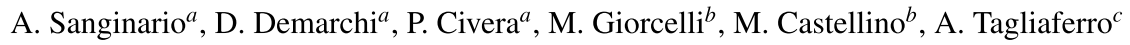 \\ ${ }^{a}$ Electronics Department, Politecnico di Torino, C.so Duca degli Abruzzi 24, 10129 Torino, Italy \\ ${ }^{b}$ Physics Department, Politecnico di Torino, C.so Duca degli Abruzzi 24, 10129 Torino, Italy \\ ${ }^{c}$ Materials Science and Chemical Engineering Department, Politecnico di Torino, C.so Duca degli Abruzzi 24, 10129 Torino, Italy
}

\begin{abstract}
The present application is based on the use of carbon nanotubes (CNTs) for biomolecular analysis using electrochemiluminescence (ECL) detection technique [1]. For this purpose we have grown self standing cylinder-shaped blocks of multi-wall CNTs (MWCNTs) by means of a catalytic chemical vapour deposition system, fed by camphor and ferrocene gases. The blocks were subsequently back-contacted and encapsulated into epoxy resin as electrical insulator and sealant, for their use as voltammetric electrodes. A ruthenium-complex solution has been used as ECL label. It has been observed a periodical light emission that lasts for hundreds of cycles, likely due to the CNTs structure. Thanks to a data-processing algorithm which exploits this behavior, the experiments show that it is possible to obtain a great increase in detection limit as compared to the common working metal electrodes (for example Au or Pt). (C) 2010 Published by Elsevier Ltd. Open access under CC BY-NC-ND license.
\end{abstract}

Keywords:

\section{Introduction}

Since their discovery [2], carbon nanotubes have attracted tremendous attention due to their extraordinary electronic, mechanical and chemical properties, which make them a strategic material for electroanalytical applications. In presented work CNTs have been considered for use in biodevices, aimed at the detection of various biomolecules [3], in order to overcome the detection limits coming from classical electrodes used in electrochemiluminescence (ECL) and cyclic voltammetry (CV) tests. ECL is a powerful electrochemical technique for measuring various biomolecules in very low quantity. Its principle is quite similar to photoluminescence (PL): to stimulate a luminescent chemical label and to readout its response in terms of emitted light. Differently from PL in which the stimuli is optic (usually a laser), in ECL the source of excitation is electric (electrodes). It is easy to understand that ECL has a big improvement in the SNR because the light collected comes only from the electrochemical label and not also from the excitation source. Moreover, required instrumentation is much less expensive and scalable to the micro and nano scale. For this purpose we have created an electrode using a millimeter long column of vertically aligned multi walls CNTs growth by chemical vapour deposition (CVD) process on patterned silicon substrates. The column was subsequently back-contacted and encapsulated with epoxy resin as electrical insulator and sealant. This electrode has been used in combination with other two electrodes, one made of gold (as counter electrode) and one made of silver (as reference electrode), in order to create an electrochemical cell as shown in fig. 1(a).

\subsection{Fabrication and technology}

Thermal-CVD process for growing multi-wall carbon nanotubes (MWCNTs) was performed at $850^{\circ} \mathrm{C}$ in a horizontal quartz tube housed in a cylindrical furnace. An inert gas flow $\left(N_{2}\right)$ carried the gas mixture of carbon precursor 

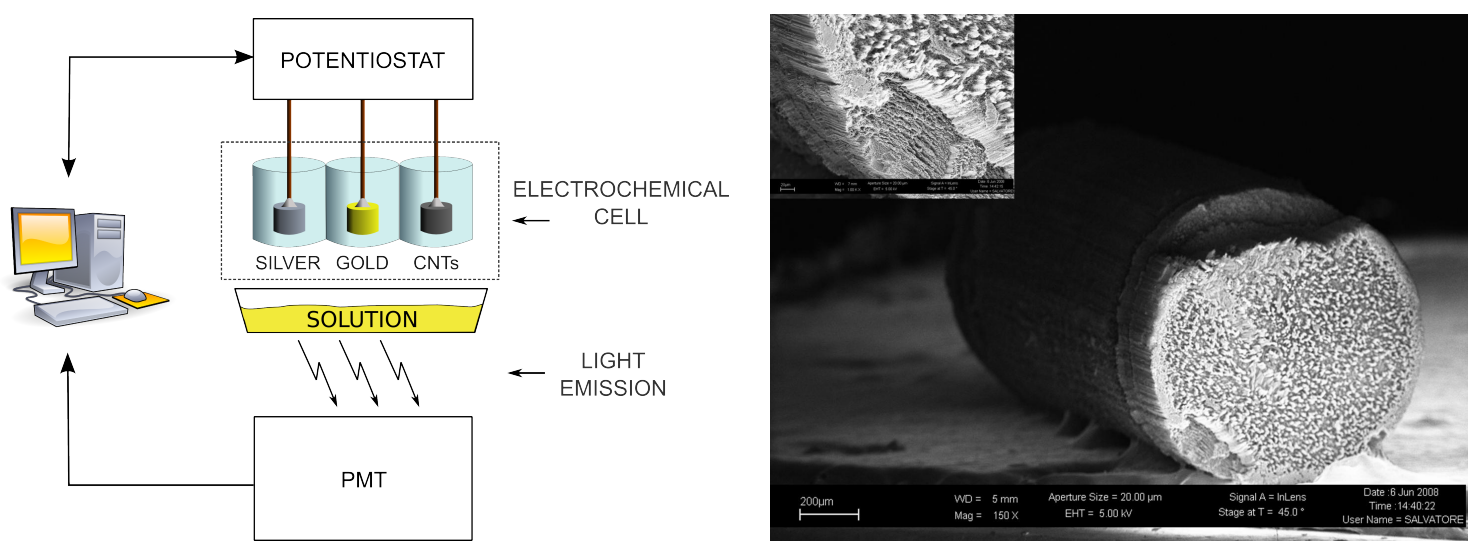

Figure 1: (a) Schematic view of the whole system: a Personal Computer controls the potentionstat for the sweep generation and a PMT collects the light generated by the electrochemical reaction; (b) Cylindrical CNTs bunch laid down on a silicon surface.

(commercial camphor) and metal catalyst (ferrocene $98 \%$ purity in weight, Aldrich) after their evaporation. The mix, in a 20/1 mass ratio (precursor/catalyst) has been carried towards the centre of the furnace, where the gases pyrolysis led to the deposition of a CNTs layer on the silicon patterned substrate. For growth parameters and details see [4] and [5]. The geometry of the cylindrical electrodes has been drawn on silicon surface using the photolithography technique. Positive photoresist (HPR 504, Fujifilm) thin layer (1.4 $\mu \mathrm{m}$ thick) has been masked, exposed to UV light and removed with its proper solution, leaving resist pillars on the silicon surface. The substrate has been subsequently covered with a double metal layer ( $5 \mathrm{~nm}$ of titanium as adhesion layer, and $100 \mathrm{~nm}$ of copper) using the thermal evaporation technique in high vacuum $\left(\sim 10^{-7}\right.$ torr). Resist has been subsequently removed with a lift-off procedure in acetone, leaving round metal-free areas of the desired geometry on the substrate, prior to CNTs deposition (fig. 1b).

\subsection{Macro-Electrode construction}

For building a manageable and mechanically stable electrode, with the correct wire contact, a copper wire or a tin connector has been pasted, with conductive glue, on one side of the resulting cylinder. Then the new electrode has been covered with epoxy resin leaving the other side free. The epoxy resin cover serves both as electrical insulator and as mechanical protection. A complete electrochemical cell was realized associating the CNT electrode (used as working electrode) to a gold electrode (used as counter electrode) and to a silver one (used as reference electrode).

\subsection{Chemicals and compositions}

Emitting solution used is based on a compound of Ruthenium, $R u(b p y)_{3}^{2+}$, Ruthenium Tris Bypiridil [6]. The ruthenium was courtesy prepared by researchers of University of Bologna. Stock solution concentration was $10^{-4} \mathrm{M}$, buffered with a phosphate solution (PBS) $10^{-1} \mathrm{M}$. In other words, a concentration of about $80 \mathrm{ng} / \mu \mathrm{l}$ of ruthenium, in which will be later dissolved tripropylamine (TPA) at its solubility limit. From this stock solution were performed many measurements at different dilutions in buffer, in order to compare sensitivity of the new system with a classical one. At this stage of the research it was detected ruthenium in solution, also if the final goal of the work will be to detect molecules attached to CNT surface, as have been done in several applications for DNA detection systems ([7], [8]). This approach was chosen because it was decided to initially test the feasibility of the device and to find the characteristics, parameters and performances of ECL detection system, then, in a second stage, experiments with DNA attached to CNT surface will be carried on.

\subsection{Electrochemical cell and complete system}

In order to manage the whole system, a custom software and a potentiostat have been built. The software, written in Python language, can manage different experiments and waveforms, while the potentiostat has been designed to generate stable cell driving potentials and to collect the very little currents from the voltammetric signal (fig. 1a). 

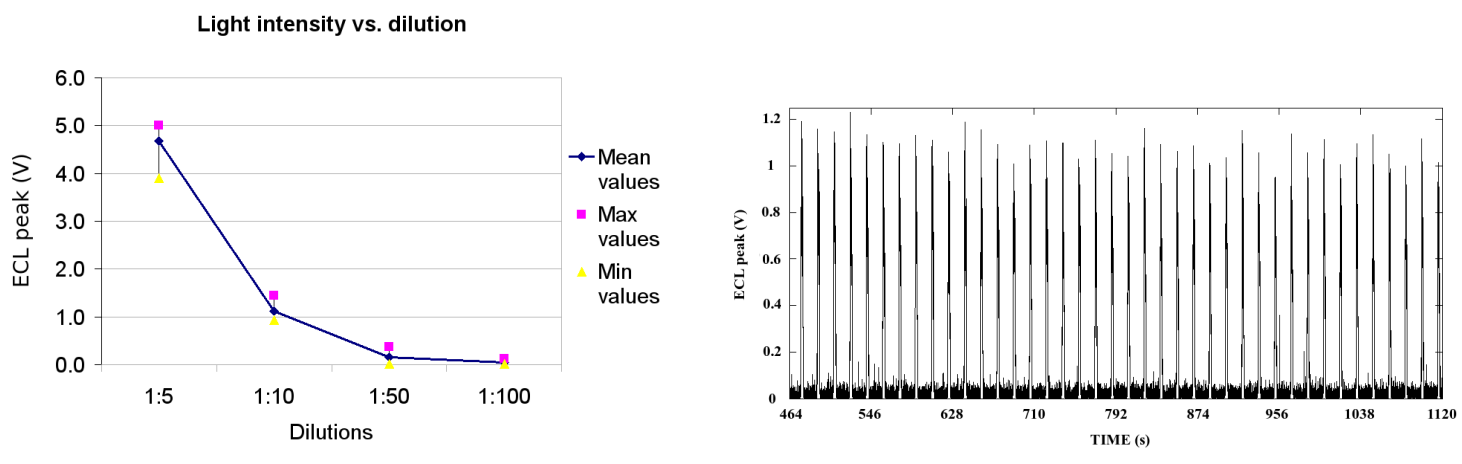

Figure 2: (a) Graph showing ECL signal against ruthenium dilutions using a standard gold working electrode; (b) Extract of some constant emission cycles of the CNTs working electrode.

At the emitting voltage of the ruthenium solution used, the chemical solution starts to emit light. For this reason the solution to be analyzed is spotted on a transparent surface on the top of an underneath photomultiplier (PMT) in order to have the maximum collection of emitted light. The PMT (an Hamamatsu H9306-03 was used for this purpose) voltage is proportional to emitted light that is itself proportional to concentration of the solution to be analyzed. In order to avoid artifacts and to increase the light detection level the whole system was placed into a dark box: a completely dark environment allows to reach best sensitivity.

\section{Results and discussion}

In order to have a direct comparison, the first measurement of the solution was done using a classical electrochemical cell with a gold working electrode. Then, the same solution was measured using the produced CNTs electrodes and the comparisons between the two setups were done. In fig. $2 \mathrm{a}$ is reported a graph were is plotted the light intensity trend over increasing dilution for a standard electrochemical cell. It is possible to notice that with gold working electrodes the detection limit is about a 1:50 dilution, in fact a 1:100 dilution signal emission is no more detectable. The results obtained by using CNTs electrode were significantly better after a proper data treatment. The improvement is mainly due not only to the highly increased contact area exhibited by the MWCNTs $\left(40-80 \mathrm{~m}^{2} / \mathrm{g}\right)$ in comparison to standard metal electrodes, but in particular to a custom algorithm which exploits a very interesting effect of CNTs electrode, discovered and recently patented [9] by some of the authors. Fig. 2b shows that the light emission with this type of electrodes is smaller compared to gold electrode but it is constant for a very large number of cycles (more than 100). We exploited this "property" by applying the concept of a common algorithm used in electronics: the averaging. By averaging over many cycles of light emission we are able to pull down the noise and consequently to extract weak light emission signals buried into the background noise. Fig. 3a shows a portion of raw data before they are processed by our algorithm. As, in this case, the concentration of Ru compound is very low, the wanted signal is buried into the noise because only few photons reached the PMT. Thanks to our data treatment it has been possible to extract the low signal from that noise thus consequently to detect very low amount of chemical analyte (fig. $3 b$ ). As said before, in our case, the detection limit of the standard electrochemical cell with a gold working electrode was a dilution of 1:50 (fig. 2a). Using CNT working electrode, associated to our customized algorithm, it was possible improve the SNR and to reach dilutions up to 1:1000 and even more, as can be seen in fig. 3b, where for a dilution of 1:1000 the signal is perfectly extracted from the noise and clearly detectable. This dilution, considering the concentration of the stock solution used, corresponds to a ruthenium concentration of $80 \mathrm{pg} / \mu \mathrm{l}$ (i.e. a $10^{-7} \mathrm{M}$ molar detection level).

\section{Conclusions and future works}

\subsection{Conclusions}

Obtained results show that CNTs can be used in ECL and voltammetry devices as electrodes, and that their use lead to an improvement in detection limit. Such electrodes present an unexpected behavior of constant signal emission 

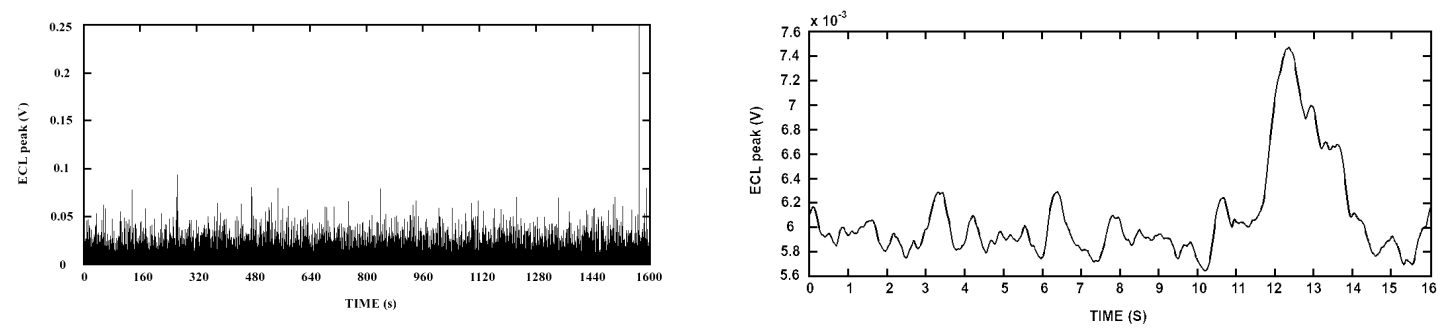

Figure 3: (a) Portion of raw data (i.e. without data treatment) in which the useful signal is buried into the noise; (b) Same portion of data shown in fig. 3a after our algorithm for a diluition of 1:1000. The signal has been extracted from the noise.

likely due to their large surface area and their disordered-matrix shape. This effect has been exploited to obtain a great improvement of the minimum signal noticeable, having a gain of at least 20 times. This improvement is essential for detection of biomolecules such as DNA or proteins and it will give the possibility to realize more efficient and precise biomedical diagnostic devices.

\subsection{Future Works}

Future work will be addressed to optimize the final setup of the system and to find its detection limit. An algorithm refinement will lead to a further improvement of the SNR. Then, experiments based on DNA and other biomolecules detection will be realized.

\section{Acknowledgements}

The authors would like to thank Dr. Leopoldo Della Ciana of Cyanagen Srl, Dr. Simone Zanarini, Prof. Francesco Paolucci of "Ciamician", University of Bologna, for their help on chemical topics and Dr. Stefano Bianco for helping in the preparation of CNTs electrodes. This work was supported by Piedmont regional project Nanocontact.

\section{References}

[1] H. Wei, E. Wang, Solid-state electrochemiluminescence of tris(2,2-bipyridyl) ruthenium, Trends in Analytical Chemistry 27 (5) (2008) $447-$ 459.

[2] S. Iijima, Helical microtubules of graphitic carbon, Nature 314 (1991) 56-58.

[3] Z. Xu, X. Chen, X. Qu, J. Jia, S. Dong, Single-wall carbon nanotube-based voltammetric sensor and biosensor, Biosensors and Bioelectronics 20 (2004) 579584.

[4] S. Musso, S. Porro, M. Rovere, A. Chiodoni, A. Tagliaferro, Physical and mechanical properties of thick self standing layers of multiwall carbon nanotubes, Diamond and Related Materials 16 (2007) 1174-1178.

[5] S. Musso, S. Porro, M. Giorcelli, A. Chiodoni, C. Ricciardi, A. Tagliaferro, Macroscopic growth of carbon nanotube mats and their mechanical properties, Carbon / Letters to the Editor 45 (2007) 1133-1136.

[6] R. D. Gerardi, N. W. Barnett, S. W. Lewis, Analytical applications of tris(2,20-bipyridyl)ruthenium(iii) as a chemiluminescent reagent, Analytica Chimica Acta 378 (1999) 1-41.

[7] H. Cai, X. Cao, Y. Jiang, P. He, Y. Fang, Carbon nano tube-enhanced electrochemical dna biosensor for dna hybridization detection, Anal. Bioanal. Chem. 375 (2003) 287- 293.

[8] J. Li, H. T. Ng, A. Cassell, W. Fan, H. Chen, Q. Ye, J. Koehne, J. Han, M. Meyyappan, Carbon nanotube nanoelectrode array for ultrasensitive dna detection, Nano letters 3 (5) (2003) 597-602.

[9] D. Demarchi, P. Civera, A. Sanginario, R. Canova, M. Turturici, L. D. Ciana, Procedimento per la generazione e l'analisi di segnali di elettrochemiluminescenza e relativo sistema (03 2010). 